\title{
Microcavity-based generation of full Poincaré beams with arbitrary skyrmion numbers
}

\author{
Wenbo Lin $\odot,{ }^{1,2, *}$ Yasutomo Ota $\odot,{ }^{3}$ Yasuhiko Arakawa, ${ }^{3}$ and Satoshi Iwamoto $\oplus^{1,2,3}$ \\ ${ }^{1}$ Research Center for Advanced Science and Technology, University of Tokyo, 4-6-1 Komaba, Meguro-ku, Tokyo 153-8505, Japan \\ ${ }^{2}$ Institute of Industrial Science, University of Tokyo, 4-6-1 Komaba, Meguro-ku, Tokyo 153-8505, Japan \\ ${ }^{3}$ Institute for Nano Quantum Information Electronics, University of Tokyo, 4-6-1 Komaba, Meguro-ku, Tokyo 153-8505, Japan
}

(Received 29 June 2020; accepted 5 April 2021; published 19 April 2021)

\begin{abstract}
A full Poincaré (FP) beam possesses all possible optical spin states in its cross section, which constitutes an optical analog of a skyrmion. Until now FP beams have been exclusively generated using bulk optics. Here we propose a generation scheme of an FP beam based on an optical microring cavity. We position two different angular gratings along with chiral lines on a microring cavity and generate an FP beam as a superposition of two light beams with controlled spin and orbital angular momenta. We numerically show that FP beams with tailored skyrmion numbers can be generated from this device, opening a route for developing compact light sources with unique optical spin fields.
\end{abstract}

DOI: 10.1103/PhysRevResearch.3.023055

\section{INTRODUCTION}

Spin angular momentum (SAM; $s$ ) and orbital angular momentum $(\mathrm{OAM} ; l)$ are fundamental parameters characterizing photons $[1,2]$ in terms of the polarization state and a helical wavefront, respectively. Recent progress in the control of SAM and OAM in optical beams has led to the generation of various vector beams with textured optical spin fields [3-6]. Among them, full Poincaré (FP) beams exhibit extremely distinctive photonic spin distributions: an FP beam possesses all possible photonic spin states in its beam cross section [7]. This unique optical spin field is receiving significant attention with potential for various applications, such as novel polarization sensing [8] and optical tweezers $[9,10]$. It is known that FP beams (and some beams with similar space-variant polarization states $[11,12])$ can be generated by superposing two light beams with opposite SAM states and different OAM states. Until now this method has been examined using bulk optics, resulting in the generation of Laguerre-Gaussian [7,13-16] and Bessel-type $[17,18]$ FP beams.

A notable property of FP beams is that their optical spin textures constitute skyrmions in two dimensions [19,20]. A skyrmion is a topological elementary excitation characterized by a topological number or a skyrmion number, $N_{\text {sk }}$. Skyrmions have been observed in various condensed-matter systems, such as chiral magnetic materials [21], Bose-Einstein condensates [22], and chiral liquid crystals [23]. Optical skyrmions and their derivatives have been observed in some optical fields, including confined modes in nanophotonic structures [24-29] as well as in FP beams [19,30]. In conjunction with the concept of optical skyrmion, FP beams offer an

\footnotetext{
*lin-w@iis.u-tokyo.ac.jp

Published by the American Physical Society under the terms of the Creative Commons Attribution 4.0 International license. Further distribution of this work must maintain attribution to the author(s) and the published article's title, journal citation, and DOI.
}

interesting area for exploring rich physics, such as the transfer of topological charges between light and matter [19] and the formation of photonic Möbius strips [31].

Until now, FP beams have been exclusively generated using bulk optics, which is not suitable for practical applications that require robustness and compactness of light sources. For unlocking the potential of FP beams in various applications and physics, it is highly desired to develop FP beam generators on chip.

Here we propose a scheme for generating an FP beam based on an optical microring cavity. Our approach utilizes a tightly confined whispering-galley mode (WGM), in which the spin-orbit interaction of light provides polarization singularities [32-34], enabling SAM-controlled light diffraction transferring the OAM into free space by the tailored perturbation of the structure. We perturb the cavity by patterning two types of angular diffraction gratings [35-37]. Accordingly, an FP beam is synthesized as a superposition of two diffracted beams with controlled SAM and OAM in the far field. We analytically show that this method in principle can produce FP beams with arbitrary $N_{\text {sk }}$, and numerically demonstrate the generation of FP beams with various $N_{\text {sk }}$ 's. Our scheme could facilitate the integration of FP beam sources with different $N_{\text {sk }}$ 's on a single chip, which may widen the application of FP beams.

\section{OPERATION PRINCIPLE}

An arbitrary polarization state or a spin state of a photon can be described using a normalized Stokes vector composed of three parameters: $\boldsymbol{s}=\left(S_{1}, S_{2}, S_{3}\right) / S_{0}$. Each parameter corresponds to an expectation value of a Pauli matrix for a photonic spin state [38]. $s$ denotes the orientation of a photonic spin, which can be visualized by a vector arrow drawn in a unit Poincaré sphere, as shown in Fig. 1(a). Figure 1(b) schematically depicts a cross-sectional Stokes vector field of an example FP beam and its projection to the surface of a unit Poincaré sphere. An FP beam possesses any possible spin 
(a)

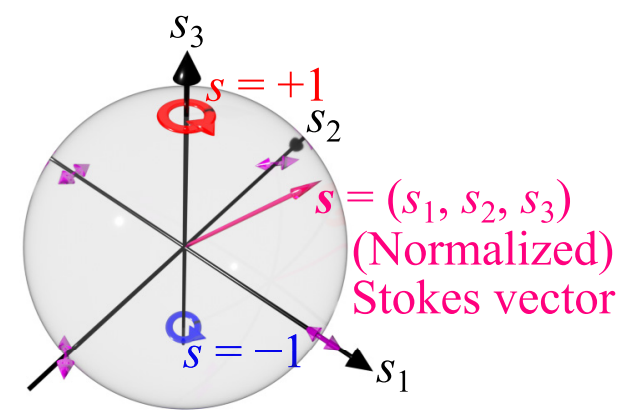

(b)

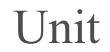

Stokes vector field

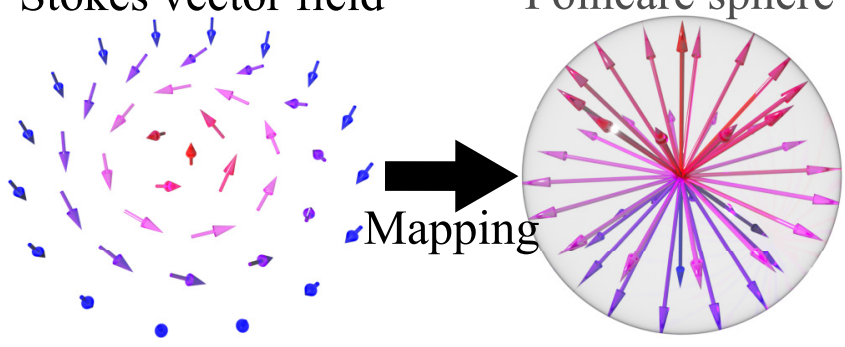

FIG. 1. (a) Unit Poincaré sphere defined in a space spanned by the normalized Stokes parameters, which represent the degree of linear polarization $\left(s_{1}\right)$, diagonal polarization $\left(s_{2}\right)$, and circular polarization $\left(s_{3}\right)$, respectively. A vector from the origin to a point on the sphere surface defines a Stokes vector and indicates the orientation of the optical spin. (b) Schematic of a cross-sectional Stokes vector field of an example FP beam and its projection to a unit Poincaré sphere.

states in the cross section, and hence, projecting them to the surface completely wraps the sphere. The topological property or the order of an FP beam is characterized by a skyrmion number that counts the number of times the photonic spins in a certain area $(A)$ wrap a unit Poincaré sphere, which is expressed as

$$
N_{\mathrm{sk}}=\frac{1}{4 \pi} \int_{A} \boldsymbol{s} \cdot\left[\partial_{x} \boldsymbol{s} \times \partial_{y} \boldsymbol{s}\right] d x d y .
$$

For an FP beam generated by superposing two beams with opposite SAMs $\left(\boldsymbol{s}_{2}=-\boldsymbol{s}_{1}\right)$ and different absolute OAMs $\left(\left|l_{2}\right| \neq\right.$ $\left.\left|l_{1}\right|\right)$, Eq. (1) yields the OAM difference, i.e., $N_{\text {sk }}=\left(l_{2}-l_{1}\right)=$ $\Delta l$. This expression is obtained by integrating Eq. (1) for a domain $A$ where the spin state of the superposed beam flips from $s_{1}$ to $-s_{1}$ (derivation can be found in Appendix C. Therefore, FP beams with any skyrmion numbers can be generated by controlling the OAMs of the beams under superposition [13,14]. The capability of generating skyrmions with $N_{\text {sk }}$ 's much larger than 1 could be of interest, because the extensively investigated magnetic skyrmions frequently exhibit $N_{\text {sk }}$ values of only 1 or $2[39,40]$.

Now we discuss our scheme of the microcavity-based generation of FP beams. We consider a microring cavity supporting a WGM rotating within the cavity. The tight spatial confinement for the propagating mode induces the spin-orbit interaction of light [32-34] and thus leads to the coupling between the spin and orbit degrees of freedom within the mode. Consequently, the WGM can be described by a superposi- tion of spin-up and spin-down components $\psi_{ \pm}$with angular momentum states of $|s, l\rangle=| \pm 1, m \mp 1\rangle$, where $m$ is the azimuthal order of the WGM and yields the total angular momentum on multiplication with $\hbar$. Figure 2(a) schematically presents the distribution of the net spin density $\left(S_{3}=\left|\psi_{+}\right|^{2}-\right.$ $\left|\psi_{-}\right|^{2}$ ) of a counterclockwise transverse electric (TE)-like WGM in a microring cavity. The spatial profiles of the spin-up and spin-down modes differ, resulting in the emergence of purely spin polarized lines, known as $C$-lines [41-43]. We utilize this phenomenon for achieving SAM-controlled light scattering from the WGM. We apply a small refractive index perturbation aligned to a $C$-line, which selectively scatters circularly polarized photons, with the handedness depending on the polarity of the $C$-line, as schematically shown in the right inset in Fig. 2(a).

By arranging a circular, periodic array of index perturbations, we can control the OAM of the scattered light according to the angular momentum conservation law expressed as $(m-s)-l=n g[35,36]$. Here $m-s$ is the effective OAM of the WGM that the angular grating feels, $l$ is the OAM of the diffracted light, $n$ is the diffraction order, and $g$ is the number of grating elements. Hereafter, we focus on the first-order diffraction of $n=1$. Figures 2(b) and 2(c) show example behaviors of the microcavity with gratings. When an angular grating with $g=m-1$ is patterned on the $C$-line of $s=+1$, a light beam described by $|s, l\rangle=|+1,0\rangle$ will be generated [Fig. 2(b)]. Meanwhile, positioning a grating of $g=m$ on the $C$-line of $s=-1$ generates a beam with $|s, l\rangle=|-1,+1\rangle$ [Fig. 2(c)].

By arranging the above two gratings in parallel, an FP beam can be synthesized in the far field as a superposition of the light beams diffracted by the two gratings, as shown in Fig. 2(d).

The far-field amplitude profile, $A(\theta, \phi)$, of a beam diffracted by one of the two angular gratings can be analytically approximated by

$$
A(\theta, \phi)=J_{|l|}\left(k d_{0} \sin \theta\right) \exp \left[i\left(l \phi-|l| \frac{\pi}{2}\right)\right],
$$

where $J_{|l|}$ is the $|l|$ th-order Bessel function of the first kind, $k$ is the wave number, $d_{0}$ is the radius of the circle where the angular grating is patterned, $\phi$ is the azimuth angle, and $\theta$ is the elevation angle $(\theta=0$ corresponds to the direction normal to the device plane). This analytical expression was derived with a toy model that assumes each grating element acts as a small electric dipole (see Appendix A). Equation (2) gives a good approximation to the profile of a diffracted beam when $\theta$ is relative small (approximately less than $30^{\circ}$ ).

\section{SIMULATION RESULTS}

As a specific design of an FP beam generator, we consider a silicon microring cavity with a radius of $3 \mu \mathrm{m}$. The waveguide of the cavity has a width of $450 \mathrm{~nm}$ and a height of $200 \mathrm{~nm}$, confining a single TE mode at the telecommunication $\mathrm{C}$ band. The refractive indices of silicon and the background environment were set to 3.4 and 1.0 , respectively. The ring cavity supports a WGM of an azimuthal order of 24 around a wavelength of $1.57 \mu \mathrm{m}$ with a large free spectral range of $\approx 30 \mathrm{~nm}$. After augmented with angular gratings as we will discuss later, the cavity mode exhibits a much lower $Q$ on the order of 


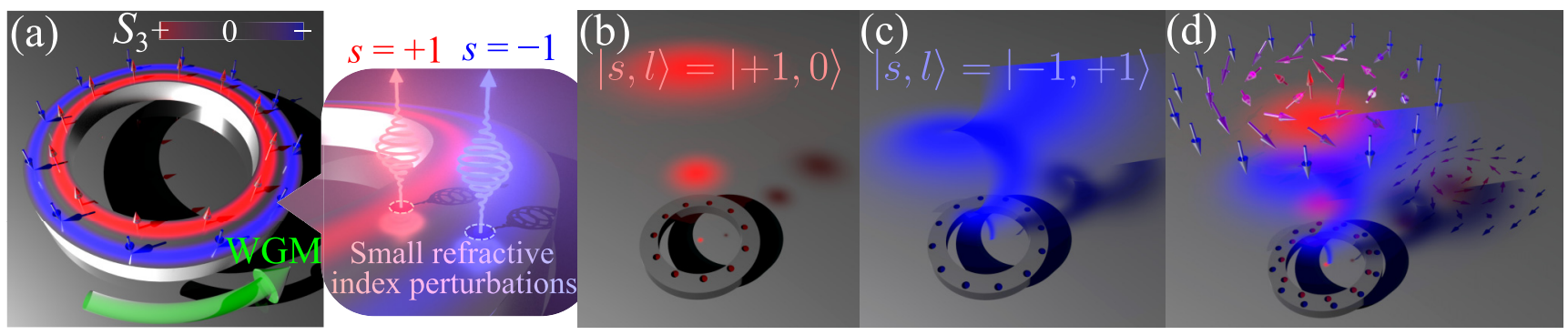

FIG. 2. (a) Schematic showing a photonic spin distribution for a counterclockwise WGM in a micoring cavity. The region with high positive (negative) spin density is indicated by red (blue). There are two singular lines with pure spin polarization of $s=+1$ or -1 , called $C$-lines. The inset shows a zoomed image of the microring, presenting light scattering due to the refractive index perturbations on the $C$-lines. The scattered light is circularly polarized, and its polarity corresponds to that of the perturbed $C$-line. (b) Light diffraction from a ring with an angular grating of $g=m-1$ on the $C$-line of $s=+1$. The diffracted light is in an angular momentum state of $|s, l\rangle=|+1,0\rangle$. (c) Same as (b) but with a grating of $g=m$ positioned on the $C$-line of $s=-1$. The diffracted light is in an angular momentum state of $|s, l\rangle=|-1,+1\rangle$. (d) Synthesis of the two diffracted light beams in (b) and (c) by simultaneously arranging the two gratings on the microring, resulting in the generation of an FP beam with an $N_{\mathrm{sk}}$ of 1 .

$10^{4}$, which indicates that the radiation from the device is dominated by that induced by the angular gratings. We focus on the counterclockwise WGM and analyze it using finite-difference time-domain (FDTD) simulations. A calculation domain of $3.5 \times 3.5 \times 1.0 \mu \mathrm{m}^{3}$ and a grid size of $10 \times 10 \times 10 \mathrm{~nm}^{3}$ were employed in the simulation. We selectively excited the mode in the simulator and continued the computation until the stationary state was reached. Figure 3(a) shows a computed mode profile of the unperturbed micoring cavity. We found $C$-lines of $s=+1$ and $s=-1$ at the positions respectively deviated by $-0.14 \mu \mathrm{m}$ and $+0.125 \mu \mathrm{m}$ from the waveguide center in the radial direction. Along each $C$-line, we arranged an array of air holes with a radius and a depth of both $40 \mathrm{~nm}$. The far fields radiated from the structure were calculated from the obtained near fields using the near-to-far field conversion technique.

First, we designed an FP beam generator emitting a beam with $N_{\text {sk }}=+1$. We patterned 23 holes on the $C$-line of $s=+1$ and 24 holes on the $C$-line of $s=-1$, so that the beams in the states of $|s, l\rangle=|+1,0\rangle$ and $|s, l\rangle=|-1,+1\rangle$ will be respectively diffracted and superposed. Figure $3(\mathrm{~b})$ presents the intensity profile of the calculated far field within an elevation angle of $\theta \leqslant 30^{\circ}$ projected onto a flat plane after collimating the beam virtually. The red dashed line in Fig. 3(b) indicates $\theta$ of $13^{\circ}$. The values of $\theta=13^{\circ}$ and $30^{\circ}$ approximately correspond to the first and second minimums in the intensity profile of the light diffracted only by the angular grating of $s=+1$ (see Appendix B). Thus, the directions of the spins are expected to be aligned downward $(s \approx-1)$ at these angles. Figure 3(c) shows the spatial distribution of the optical spin field within $\theta \leqslant 13^{\circ}$. Spins are directed upward near the center, rotate with changing azimuth angle, and gradually flip their direction to downward as the domain edge is approached. Figure 3(d) plots a projection of the spin texture to a unit Poincaré sphere. The full coverage of the surface demonstrates that the generated beam is indeed an FP beam. The projection map suggests that the observed optical spin field forms a Bloch-type skyrmion. We note that other types of skyrmions, such as the Néel-type one can be generated by rotating one of the angular gratings with respect to the other (see Appendix F).
Subsequently, we calculated $N_{\text {sk }}$ according to Eq. (1). The integration was performed from the center to a particular $\theta$ and for all $\phi$. Figure 3(e) displays the $\theta$ dependence of $N_{\mathrm{sk}}$. At $\theta \approx 13^{\circ}, N_{\text {sk }}$ reaches a near unity value of 0.98 , which compares well with the designed value of $N_{\mathrm{sk}}=+1$. The
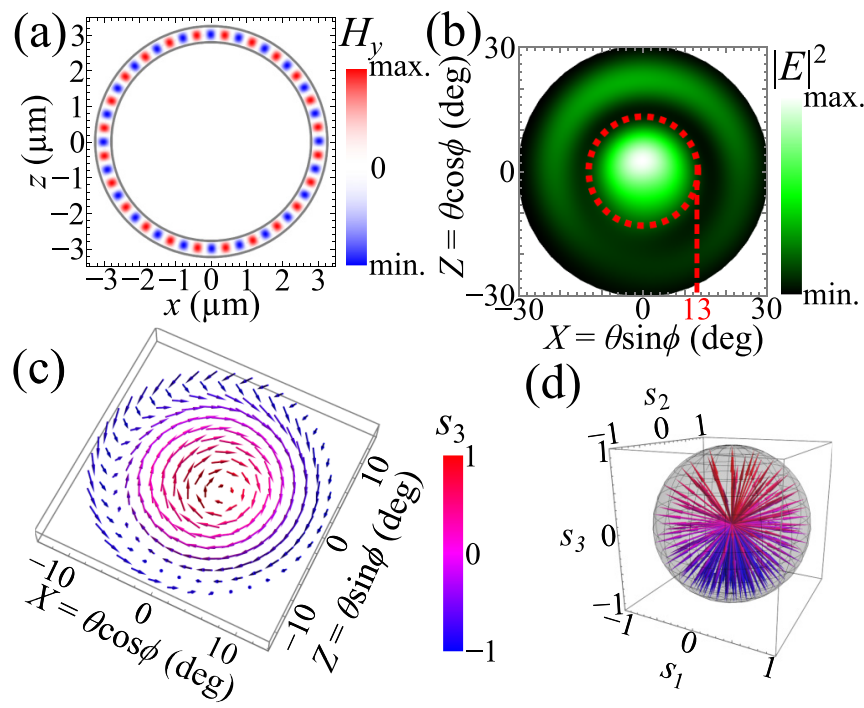

(d) (deg)
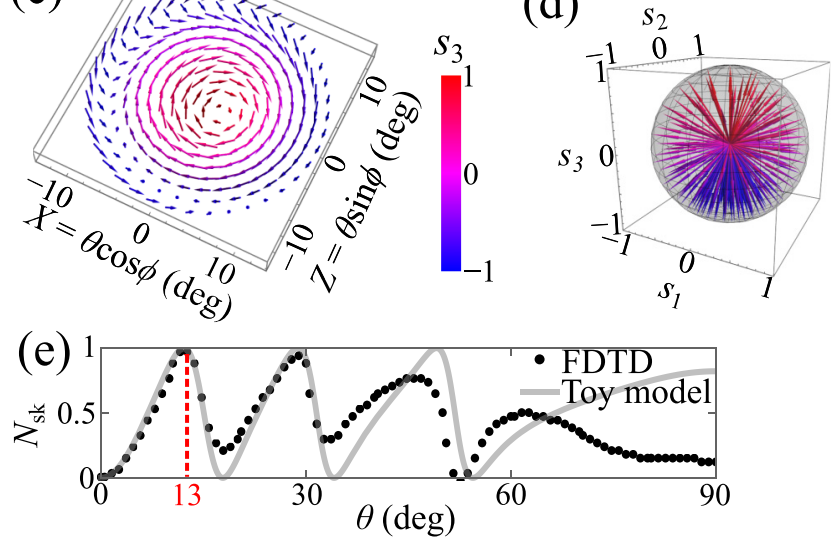

FIG. 3. (a) Calculated mode profile of a counterclockwise TElike WGM with an azimuthal order of 24 . The out-of-plane magnetic field is shown. (b) Projected far-field intensity when two angular gratings of $g=23$ and 24 are applied along the $C$-lines of $s=+1$ and $s=-1$, respectively. Here we considered the collimation of the far field and its projection to a flat plane. The conversion to the Cartesian coordinates in the flat plane $(X, Z)$ can be achieved by multiplying an appropriate scale factor to the coordinates defined using the elevation $(\theta)$ and the azimuth $(\phi)$ angles. (c) Stokes vector field within $\theta=13^{\circ}$. (d) Stokes vector of (c) projected to a unit Poincaré sphere. (e) Skyrmion number $N_{\text {sk }}$ versus elevation angle $\theta$. 
remaining error of 0.02 may be caused by the imperfect circular polarization of the scattered light, resulting from the finite size of the scatter and/or from the multiple reflection of the scattered light before exiting the structure. We also analytically deduced values of $N_{\text {sk }}$ using our toy model and overlaid the result on the plot in Fig. 3(e). The analytical curve explains well the FDTD results, in particular when $\theta$ is small, in which case the toy model can be regarded as a good approximation. For both the FDTD and analytical results, we observed oscillations in $N_{\text {sk }}$ for $\theta>13^{\circ}$. The maxima and minima of the oscillations correspond to the point where the intensity of one of the two scattered beams under superposition approaches zero. Analytically, $N_{\text {sk }}$ should oscillate between 0 and the designed $N_{\text {sk }}$, leading to the generation of a skyrmion and an antiskyrmion alternately. This skyrmionic structure is known as a skyrmion multiplex [44], the simplest case of which is known as skyrmionium. Another skyrmion state with $N_{\mathrm{sk}}=1 / 2$, called half skyrmion or meron [45], could also be produced by spatially filtering the beam by a diaphragm passing only $\theta<8^{\circ}$.

Next, we synthesized an FP beam with $N_{\text {sk }}=-1$ or an antiskyrmionic beam. For this, $g$ of the grating on the $C$-line of $s=-1$ was changed from 24 to 26 , so that a beam in a state of $|s, l\rangle=|-1,-1\rangle$ will be radiated from the grating. Figures 4(a) and 4(b), respectively, show the computed far-field intensity profile and the corresponding optical spin field within $\theta=13^{\circ}$. The latter texture shows an antivortex behavior as expected. The calculated $N_{\text {sk }}$ reaches -1.07 at $\theta=13^{\circ}$, as presented in Fig. 4(c). These results demonstrate that an antiskyrmionic FP beam can also be generated within our scheme. Finally, we present an example device generating an FP beam with a large $N_{\text {sk }}$ of -5 . For this demonstration, $g$ of the grating on the $C$-line of $s=-1$ was increased to 30 , which radiates a beam in the state of $|s, l\rangle=|-1,-5\rangle$. Figures 4(d) and 4(e) show the computed far-field intensity profile and the corresponding spin field, respectively. From the latter plot, it can be observed that the optical spins wind five times around the field center. Figure 4(f) displays the $\theta$ dependence of the calculated $N_{\mathrm{sk}}$. The minimum $N_{\mathrm{sk}}$ of -5.22 was found at $\theta=30^{\circ}$, demonstrating an FP beam with a large $N_{\mathrm{sk}}$ of -5 . Meanwhile, for the toy model, $N_{\mathrm{sk}}$ should reach -5 close to $\theta=13^{\circ}$ [indicated by cyan broken lines in Figs. 4(d)4(f)]. However, we did not observe an $N_{\text {sk }}$ of approximately -5 at $\theta=13^{\circ}$ in the FDTD simulations. This unexpected behavior in the computation model seemingly arises from the weak diffracted beam in the state of $|s, l\rangle=|-1,-5\rangle$ close to $\theta=13^{\circ}$, which can be confirmed in the corresponding far-field pattern displayed in Appendix B. The weak signal is obscured by the background noise in the simulator and, thus, yields an unpredictable result. Here the large $l$ of the beam induces the weak signal at small $\theta$ values. More robust generation of FP beams with high $N_{\mathrm{sk}}$ 's could be possible by engineering the size and/or shape of the microring and the pattern of the far fields (see Appendix G).

\section{CONCLUSION}

In summary, we numerically demonstrated FP beam generation from a microring cavity. We augmented the microring by angular gratings patterned on the $C$-lines, which diffracted (a)

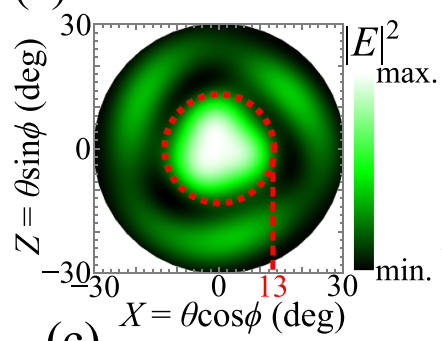

(b)

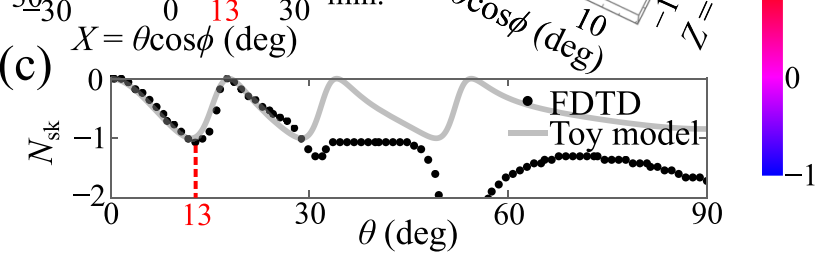

(d)

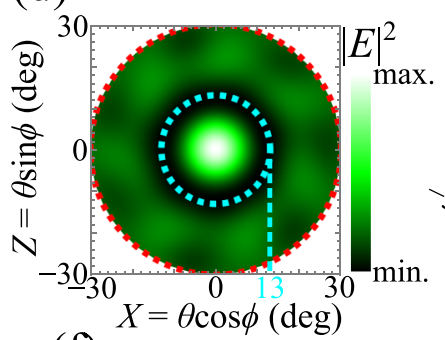

(e)

(f)

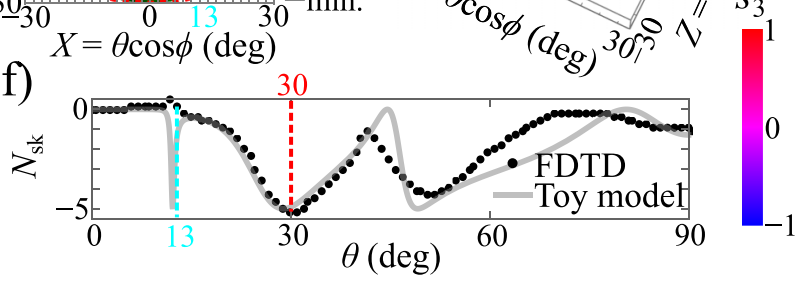

FIG. 4. Generation of FP beams with different $N_{\text {sk }}$ 's. (a)-(c) Beam properties of the device designed for generating an FP beam with $N_{\text {sk }}$ of -1 : (a) projected far-field intensity profile, (b) Stokes vector field, and (c) calculated skyrmion number versus elevation angle. (d)-(f) Same as (a)-(c) but for the device designed with $N_{\text {sk }}$ of -5 .

SAM-controlled beams with different OAMs. We showed that the superposition of the beams from the microcavity results in an FP beam with an arbitrary controllable $N_{\text {sk }}$. We examined concrete designs of silicon-based microring cavities and verified the generation of FP beams with various $N_{\text {sk }}$ 's. We believe that the compact FP beam generators could find broad applications in optical communication and optical sensing and play a significant role in exploring condensed matter physics.

\section{ACKNOWLEDGMENTS}

This research was supported by JST CREST Grant No. JPMJCR19T1 and JSPS KAKENHI Grants No. JP15H05700, No. JP17H02796, and No. JP19J13955.

\section{APPENDIX A: A TOY MODEL FOR DESCRIBING DIFFRACTION PATTERNS}

We develop a toy model consisting of a circular array of radiative dipoles for analytically deriving diffraction patterns of angular diffraction gratings interacting with a whisperinggalley mode (WGM). We consider infinitesimal dipoles, the respective polarization of which emulates the local optical 
polarization of the WGM. We assumed that other scattering mechanisms occurring in the microring cavity are negligible and solely considered the radiation from the dipoles located equidistantly. The radiation from one of the dipoles can be written using a well-known approximated form given by

$$
\boldsymbol{E}_{\text {far }, \text { single }}(\boldsymbol{r}) \propto \frac{\boldsymbol{r}-\boldsymbol{r}_{\mathbf{0}}}{\left|\boldsymbol{r}-\boldsymbol{r}_{\mathbf{0}}\right|} \times\left[\frac{\boldsymbol{r}-\boldsymbol{r}_{\mathbf{0}}}{\left|\boldsymbol{r}-\boldsymbol{r}_{\mathbf{0}}\right|} \times \boldsymbol{E}_{\mathrm{WGM}}\left(\boldsymbol{r}_{0}\right)\right] \frac{e^{i k\left|\boldsymbol{r}-\boldsymbol{r}_{\mathbf{0}}\right|}}{\left|\boldsymbol{r}-\boldsymbol{r}_{\mathbf{0}}\right|},
$$

where $\boldsymbol{r}_{0}$ is the position of the dipole and $k$ is the wave number. The source dipole moment is replaced by a local electric field vector of the WGM, $\boldsymbol{E}_{\mathrm{WGM}}\left(\boldsymbol{r}_{0}\right)$, so as to express the scattered radiation of the mode by a grating element at $\boldsymbol{r}_{0}$. In far-field region $\left(k r \gg 1\right.$ and $\left.r \gg r_{0}\right)$, Eq. (A1) can be further approximated to a spherical wave:

$$
\begin{aligned}
\boldsymbol{E}_{\mathrm{far}, \text { single }}(\boldsymbol{r}) & \sim \frac{\boldsymbol{r} \times\left[\boldsymbol{r} \times \boldsymbol{E}_{\mathrm{WGM}}\left(\boldsymbol{r}_{0}\right)\right]}{r^{2}} \frac{e^{i k r}}{r} \exp \left[-i k \frac{\boldsymbol{r} \cdot \boldsymbol{r}_{0}}{r}\right] \\
& =\boldsymbol{P}_{\mathrm{far}, \text { single }}(\phi, \theta) R(r) \tilde{E}_{\mathrm{far}, \text { single }}(\phi, \theta) .
\end{aligned}
$$

In the last equal of this equation, we decomposed the far field into three components. The first factor, $\boldsymbol{P}_{\text {far, single }}(\phi, \theta)=\boldsymbol{r} \times$ $\left[\boldsymbol{r} \times \boldsymbol{E}_{\mathrm{WGM}}\right] / r^{2}$, represents the vectorial nature of the field. The set of the cross products, $\boldsymbol{r} \times \boldsymbol{r} \times$, projects the polarization of the source dipole field (which equals $\boldsymbol{E}_{\text {WGM }}$ ) to the plane normal to the wave vector (or to the vector $\boldsymbol{r}$ ). The second factor, $R(r)=e^{i k r} / r$, expresses the propagation of a scalar spherical wave. The third factor, $\tilde{E}_{\text {far, single }}(\phi, \theta)=$ $\exp \left[-i k \boldsymbol{r} \cdot \boldsymbol{r}_{0} / r\right]$, is also a scalar part of the far field. The last two factors merely describe the field intensity distribution in the far field and do not scramble the light polarization. Therefore, the polarization distribution can be solely represented by $\boldsymbol{P}_{\text {far,single }}(\phi, \theta)$. We evaluate the vector part or the light polarization on a coordinate described by

$$
\left(\begin{array}{c}
E_{R} \\
E_{X} \\
E_{Y}
\end{array}\right)=\left(\begin{array}{ccc}
1 & 0 & 0 \\
0 & \cos \phi & -\sin \phi \\
0 & \sin \phi & \cos \phi
\end{array}\right)\left(\begin{array}{c}
E_{r} \\
E_{\theta} \\
E_{\phi}
\end{array}\right)
$$

$E_{X}$ and $E_{Y}$ components correspond to the light polarization in the collimated far field. Then $\boldsymbol{P}_{\text {far,single }}$ can be expressed as

$$
\boldsymbol{P}_{\text {far, single }}(\phi, \theta)=-\left(\begin{array}{c}
0 \\
E_{\mathrm{WGM}, x} \\
E_{\mathrm{WGM}, y}
\end{array}\right)+\left(\begin{array}{c}
E_{\mathrm{WGM}, x} \\
E_{\mathrm{WGM}, y} \\
E_{\mathrm{WGM}, z}
\end{array}\right) \cdot\left(\begin{array}{c}
(1-\cos \theta) \cos \phi \\
(1-\cos \theta) \sin \phi \\
\sin \theta
\end{array}\right)\left(\begin{array}{c}
0 \\
\cos \phi \\
\sin \phi
\end{array}\right) .
$$

The second term in the right-hand side becomes negligible as far as both $E_{z}$ and $\theta$ are small. These conditions match with our case, where light scattering from a TE mode $\left(E_{z} \approx 0\right)$ is considered mainly for $\theta$ of no more than $30^{\circ}$. We note that, even at $\theta=30^{\circ}$, radiation from a circular dipole exhibits a high degree of circular polarization of $\left|s_{3}\right|=\left|S_{3} / S_{0}\right|=$ $-2 \operatorname{Im}\left[E_{Y}{ }^{*} E_{X}\right] /\left(E_{X}{ }^{*} E_{X}+E_{Y}{ }^{*} E_{Y}\right) \approx 0.99$ in the far field, well sustaining the one-to-one correspondence between the polarization of a local source dipole and that of the collimated far field. These discussions render an approximated equation, $\boldsymbol{P}_{\text {far,single }} \sim-\left(0, E_{\mathrm{WGM}, \mathrm{x}}, E_{\mathrm{WGM}, \mathrm{y}}\right)$ for small $\theta$.

Now we discuss a far field created by an array of the scattering elements. Considering the phase difference of radiation among the grating elements, the superposed radiation field can be expressed as

$$
\begin{aligned}
\boldsymbol{E}_{\mathrm{far}}(\boldsymbol{r}) \sim & \sum_{j=0}^{g-1} \frac{\boldsymbol{r}-\boldsymbol{r}_{\boldsymbol{j}}}{\left|\boldsymbol{r}-\boldsymbol{r}_{\boldsymbol{j}}\right|} \\
& \times\left(\frac{\boldsymbol{r}-\boldsymbol{r}_{\boldsymbol{j}}}{\left|\boldsymbol{r}-\boldsymbol{r}_{\boldsymbol{j}}\right|} \times \boldsymbol{E}_{\mathrm{WGM}, j} \exp \left[i m 2 \pi \frac{j}{g}\right]\right) \frac{e^{i k\left|\boldsymbol{r}-\boldsymbol{r}_{j}\right|}}{\left|\boldsymbol{r}-\boldsymbol{r}_{\boldsymbol{j}}\right|} .
\end{aligned}
$$

Here $g$ is the number of grating elements. $\boldsymbol{r}_{j}=$ $d_{0}(\cos [2 \pi j / g], \sin [2 \pi j / g], 0)$ is the position of the $j$ th grating element. The grating elements are arranged with equal intervals on a circle in the $x-y$ plane having a center $(0,0,0)$ and a radius $d_{0} . \boldsymbol{E}_{\mathrm{WGM}, j}$ is the polarization vector of the WGM at $\boldsymbol{r}_{j} . m$ is the azimuthal order of the WGM. Following the discussion about the single dipole, the vector part of Eq. (A5) also sustains the one-to-one correspondence with the polarization of the local source dipole for small $\theta$. This means that the polarization distribution becomes homogeneous within the approximation if all the grating elements scatter light with the same polarization. Here we assume that $\boldsymbol{E}_{\mathrm{WGM}, j}$ is a circular polarization with a spin angular momentum (SAM) of $s$. Then $\boldsymbol{E}_{\mathrm{WGM}, j}$ can be rewritten to $\boldsymbol{E}_{\mathrm{WGM}, 0} \exp [-i s 2 \pi j / g]$, where $\boldsymbol{E}_{\mathrm{WGM}, 0}$ is the polarization vector of the WGM at $\left(d_{0}, 0,0\right)$. Taking into account this additional phase factor of $\exp [-i s 2 \pi j / g]$, together with $\exp [i m 2 \pi j / g]$ in Eq. (A5), the $r$-independent scalar part of Eq. (A5), $\tilde{E}_{\mathrm{far}}$, can be written as

$$
\begin{aligned}
\tilde{E}_{\mathrm{far}}(\phi, \theta) \propto & \frac{1}{g} \sum_{j=0}^{g-1} \exp \left[-i \rho \cos \left(2 \pi \frac{j}{g}-\phi\right)\right] \\
& \times \exp \left[i(m-s) 2 \pi \frac{j}{g}\right]
\end{aligned}
$$

where $\rho=k \sin \theta d_{0}$. This equation takes a form of inverse discrete Fourier transform. We consider the limit of infinite $g$ and obtain a continuous Fourier transform given by

$$
\begin{aligned}
\tilde{E}_{\text {far }}(\phi, \theta) \stackrel{g \rightarrow \infty}{\longrightarrow} & \sum_{n=-\infty}^{\infty} \frac{1}{2 \pi} \int_{0}^{2 \pi} \exp [-i \rho \cos (\xi-\phi)] \\
& \times \exp \left[i l_{n} \xi\right] d \xi
\end{aligned}
$$

where $l_{n}=m-s-n g$ is the orbital angular momentum (OAM) order of the diffracted light of $n$ th-order diffraction. The integral in Eq. (A7) constitutes an inverse Fourier transform of a so-called perfect optical vortex with an OAM of $l_{n}$ and is known to be a $l_{n}$ th order Bessel function of the first 
(a)

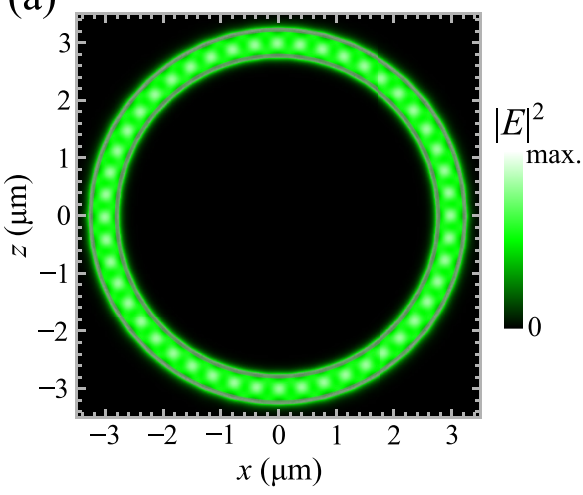

(b)

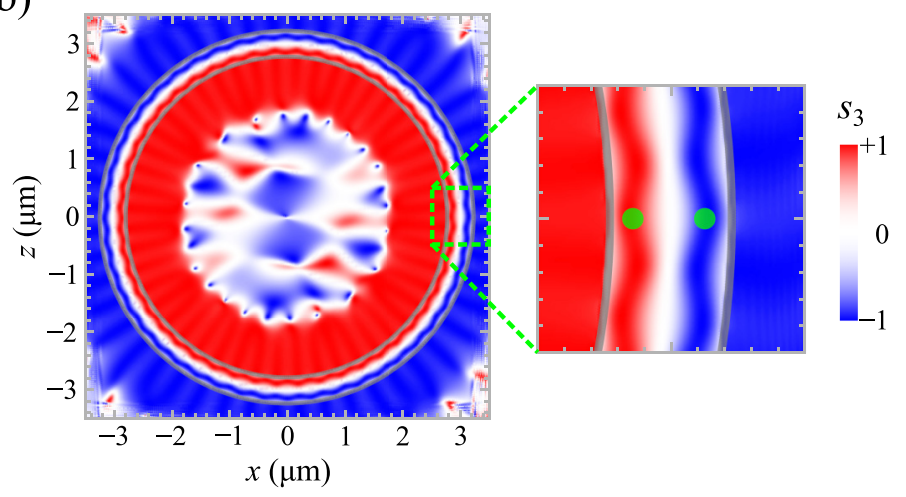

FIG. 5. Calculated counterclockwise WGM of the azimuthal order of 24 for the unperturbed microring cavity: (a) field intensity profile; (b) spatial distribution of the degree of circular polarization, which equals to the normalized Stokes parameter $s_{3}$. The right inset is a magnified view of the area surrounded by the green dashed line. The green dots in the inset show the radial positions and dimensions of the grating elements on the $C$-lines.

kind, $J_{l_{n}}(\rho)[46,47]$. Indeed, we can show that Eq. (A7) can be interpreted as a Fourier transform of the generating function of $J_{l_{n}}(\rho)$. The generating function is expressed as

$$
\exp \left[\frac{\rho}{2}\left(t-t^{-1}\right)\right]=\sum_{l_{n}=-\infty}^{\infty} J_{l_{n}}(\rho) t^{l_{n}}
$$

By replacing $t \rightarrow-i e^{i \xi}$, we get a Fourier series

$$
\exp [-i \rho \cos \xi]=\sum_{l_{n}=-\infty}^{\infty} J_{l_{n}}(\rho) \exp \left[-i l_{n} \frac{\pi}{2}\right] e^{i \xi l_{n}}
$$

which yields

$$
\frac{1}{2 \pi} \int_{0}^{2 \pi} \exp [-i \rho \cos \xi] \exp \left[i l_{n} \xi\right] d \xi=J_{l_{n}}(\rho) \exp \left[-i l_{n} \frac{\pi}{2}\right] \text {. }
$$

This equation has the same form with the integral in Eq. (A7). By substituting Eq. (A10) into Eq. (A7), we finally obtain

$$
\tilde{E}_{\mathrm{far}}(\phi, \theta) \propto \sum_{n=-\infty}^{\infty} J_{\left|l_{n}\right|}\left(k d_{0} \sin \theta\right) \exp \left[i\left(l_{n} \phi-\left|l_{n}\right| \frac{\pi}{2}\right)\right] .
$$

This equation gives a good approximation to Eq. (A6) when the spatial sampling rate or $g$ is large and when the spatial frequency or $\theta$ is small. These conditions also correspond with the situation discussed in the main text, where the first-order diffraction is treated and the dominant radiation is concentrated to the region with small $\theta$ as far as $m \approx g$.

\section{APPENDIX B: DETAILED FDTD RESULTS}

In this section, we discuss more details of the results computed by the finite-difference time-domain (FDTD) method. Figures 5(a) and 5(b), respectively, show the calculated intensity distribution and photonic spin density distribution $\left[s_{3}=-2 \operatorname{Im}\left[E_{z}{ }^{*} E_{x}\right] /\left(E_{x}{ }^{*} E_{x}+E_{z}{ }^{*} E_{z}\right)\right]$ of a WGM without gratings. Here, we computed a TE-like counterclockwiserotating WGM with the azimuthal order of 24 . The intensity distribution is nearly constant in the azimuthal direction but with slight modulation. The wavy pattern is induced by the intrusion of the counter-rotating WGM, which was weakly excited in the simulator due to the imperfect mode selection. The similar distribution can be found in the spin density distribution, which shows two wavy lines with mutually opposite polarity. The spin-up (-down) region is found near the inner (outer) edge of the microring, which is very clearly displayed in the inset of Fig. 5(b). The light-green dots show the radial positions of the grating elements. They are placed above the $C$-lines.

We computed far fields based on the near-to-far field conversion method, using near fields recorded at a plane just above the microring cavity. Figures 6(a)-6(d) show computed far-field patters for a perturbed ring cavity with a 23-element grating on the $C$-line of $s=+1$, which is designed to diffract a beam in a state of $|s, l\rangle=|+1,0\rangle$. The intensity profile plotted in Fig. 6(a) exhibits a circularly symmetric beam pattern. Figure 6(b) shows a phase profile of the diffracted beam. In the azimuth direction, the phase is almost constant, which corresponds to an OAM state of $l=0$. Figure 6(c) shows the spatial distribution of the spin density distribution $\left[s_{3}=\right.$ $\left.-2 \operatorname{Im}\left[E_{Z}^{*} E_{X}\right] /\left(E_{X}{ }^{*} E_{X}+E_{Z}{ }^{*} E_{Z}\right)\right]$. Nearly pure and homogeneous spin-up polarization is observed across the entire far field, suggesting the successful radiation of circularly polarized light from the $C$-line. The minor blue regions, where the spin state is not pure spin-up anymore, occur in the places where the far-field intensity itself is very weak. We consider that, at these regions in the far field, unspecified background noise in the simulator causes the unexpected spin flip. Figure 6(d) shows a slice of the far field along with the line of $Z=0$. For comparison, we also plot the result obtained by the toy model. The two lines results agree well within the range of $\theta \leqslant 30^{\circ}$.

Figures $6(\mathrm{e})-6(\mathrm{~h})$ show the same set of results with those depicted in Figs. 6(a)-6(d) but of the device with a 24element grating on the $C$-line of $s=-1$, which is designed to diffract a beam in a state of $|s, l\rangle=|-1,+1\rangle$. The intensity plot shows a doughnut-like distribution around the far-field center. Together with this result, the phase distribution plot demonstrates that the far field carry OAM of $l=-1$. The spin density distribution shows that the dominant spin state is $s=-1$. Again, the unwanted spin flip occurs in the region 

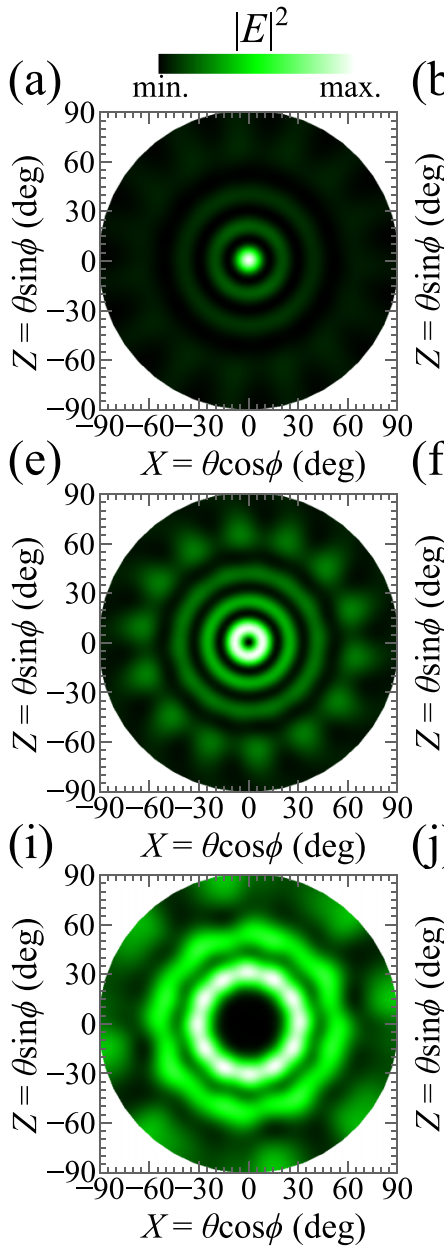
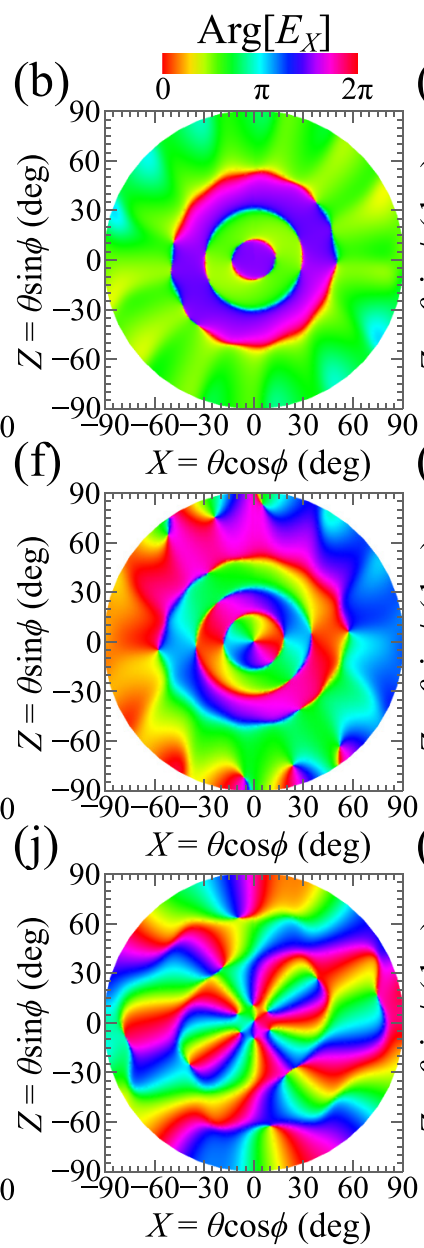
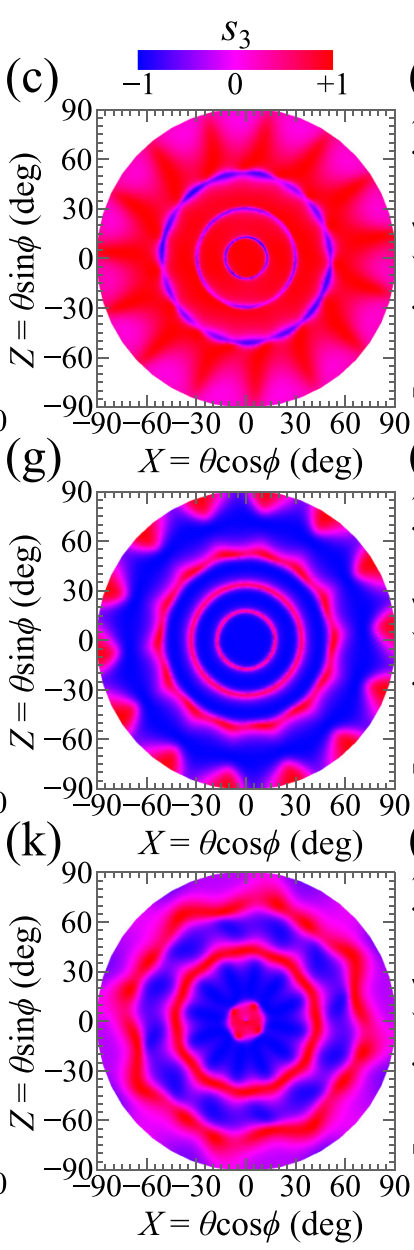
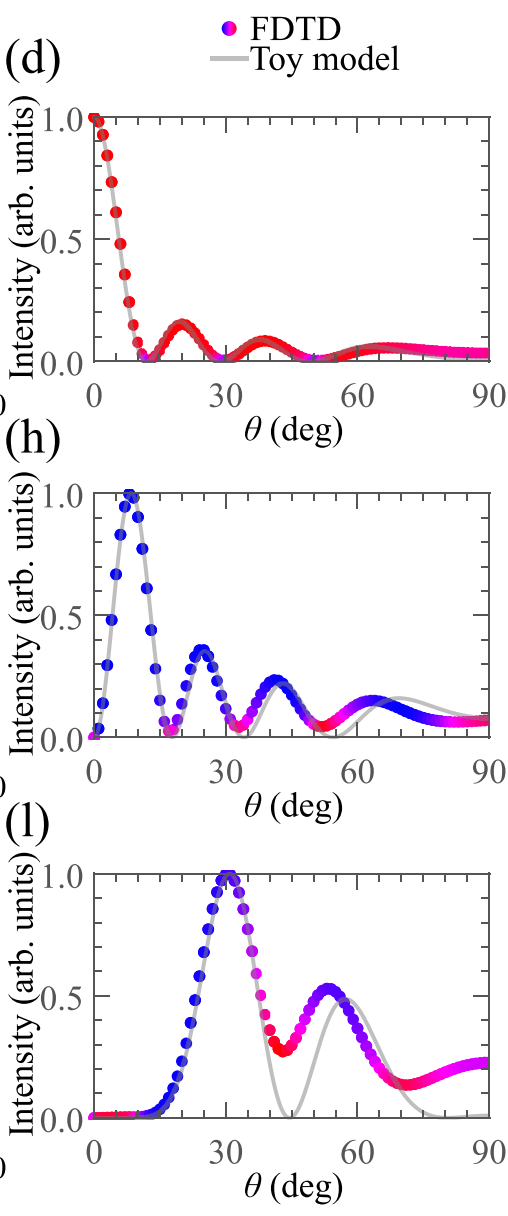

FIG. 6. (a)-(d) Far field of the light diffracted by the angular grating with 23 grating elements on the $s=+1 C$-line: (a) Projected intensity profile, (b) phase distribution of the projected electric field $E_{X}$, (c) photonic spin density distribution, (d) field intensity plot along with the line of $Z=0$. The color of the dot reflects the local spin state. The gray solid line shows the corresponding result obtained by the toy model. (e)-(h) Same as (a)-(d) but computed for the device with an angular grating of $g=24$ on the $C$-line of $s=-1$. (i) - (l) Same as (e)-(h) but with a grating of $g=30$.

with weak far-field intensity, which is more visible in the current case. Nevertheless, we confirmed a good agreement between the FDTD and the toy models as in Fig. 6(h).

The same calculations were also performed for the device with a 30-element grating on the $C$-line of $s=-1$, as plotted in Figs. 6(e)-6(h). In this case, the far field in the state of $|s, l\rangle=|-1,-5\rangle$ will be diffracted from the cavity. The intensity plot exhibits an even wider doughnut pattern in the far field, which was confirmed to carry OAM of $l=-5$ in conjunction with the plot of the phase pattern. The spin distribution exhibits the unwanted spin flip even near the far-field center $\left(\theta \approx 0^{\circ}\right)$. This unexpected result arises from further weaker field intensity around the field center owing to the widened hole in the doughnut beam with the increased $|l|$. Even in this situation, the FDTD result agrees well with the toy mode when $\theta<30^{\circ}$.

\section{APPENDIX C: ANALYTICAL SKYRMION NUMBER FOR AN FP BEAM}

Here we analytically derive an expression for a skyrmion number of an FP beam generated by superposing two vortex beams with different OAM and opposite SAM values. We use a Jones vector $\left|s_{1}\right\rangle$ for expressing a polarization state. $\left|s_{1}\right\rangle$ is defined in a $x-y$ plane and can be fully described with two parameters: an amplitude ratio $(\tan \alpha)$ and a phase difference $(\delta)$ between the $x$ and $y$ electric field components. Then $\left|s_{1}\right\rangle$ is expressed as

$$
\left|s_{1}\right\rangle=\left(\begin{array}{c}
\cos \alpha \\
e^{i \delta} \sin \alpha
\end{array}\right) .
$$

The opposite (or orthogonal) polarization state $\left|s_{2}\right\rangle$ is written as

$$
\left|s_{2}\right\rangle=\left(\begin{array}{c}
-\sin \alpha \\
e^{i \delta} \cos \alpha
\end{array}\right),
$$

which satisfies $\left\langle s_{2} \mid s_{1}\right\rangle=0$. The polarization state $\left|s_{1}\right\rangle$ can also be described using a three-component Stokes vector

$$
\boldsymbol{s}_{1}=\left(\begin{array}{c}
\cos 2 \alpha \\
\sin 2 \alpha \cos \delta \\
\sin 2 \alpha \sin \delta
\end{array}\right),
$$

and the Stokes vector for the orthogonal state is $\boldsymbol{s}_{2}=-\boldsymbol{s}_{1}$. Meanwhile, the other angular momentum, orbital angular 
momentum (OAM), can be characterized by a spiral wavefront. It is known that the mode cross section of an optical beam carrying a well-defined OAM is circularly symmetric in general, and the spiral wavefront can be written as

$$
\psi(r, \phi)=A(r) e^{i[l \phi+\gamma(r)]},
$$

where $r$ is the radial distance from the beam center, $\phi$ is the azimuth angle, $A$ is the amplitude, $l$ is the order of OAM, and $\gamma$ is the phase offset.
Accordingly, the polarization state of an FP beam generated as the superposition of two different OAM beams with opposite SAMs can be written as

$$
\begin{aligned}
\psi(r, \phi)= & A_{1}(r) e^{i\left[l_{1} \phi+\gamma_{1}(r)\right]}\left(\begin{array}{c}
\cos \alpha \\
e^{i \delta} \sin \alpha
\end{array}\right) \\
& +A_{2}(r) e^{i\left[l_{2} \phi+\gamma_{2}(r)\right]}\left(\begin{array}{c}
-\sin \alpha \\
e^{i \delta} \cos \alpha
\end{array}\right),
\end{aligned}
$$

and the normalized Stokes vector deduced from $\psi$ is given by

$$
\boldsymbol{s}=\frac{A_{1}^{2}-A_{2}^{2}}{A_{1}^{2}+A_{2}^{2}} s_{1}+\frac{2 A_{1} A_{2}}{A_{1}^{2}+A_{2}^{2}} \times\left(\begin{array}{c}
\sin 2 \alpha \cos (\Delta l \phi+\Delta \gamma) \\
\cos 2 \alpha \cos (\Delta l \phi+\Delta \gamma) \cos \delta-\sin (\Delta l \phi+\Delta \gamma) \sin \delta \\
\cos 2 \alpha \cos (\Delta l \phi+\Delta \gamma) \sin \delta+\sin (\Delta l \phi+\Delta \gamma) \cos \delta
\end{array}\right)
$$

where $\quad \Delta l=l_{2}-l_{1} \quad$ and $\quad \Delta \gamma(r)=\gamma_{2}(r)-\gamma_{1}(r)$. The skyrmion number of a certain region $A=$ $\left\{(r, \phi) \mid r_{1} \leqslant r \leqslant r_{2}, 0 \leqslant \phi \leqslant 2 \pi\right\}$ is then given by

$$
\begin{aligned}
N_{\mathrm{sk}} & =\frac{1}{4 \pi} \iint_{A} \boldsymbol{s} \cdot\left[\partial_{x} \boldsymbol{s} \times \partial_{y} s\right] d x d y \\
& =\frac{1}{4 \pi} \iint_{A} \boldsymbol{s} \cdot\left[\partial_{r} \boldsymbol{s} \times \partial_{\phi} s\right] d r d \phi \\
& =\frac{1}{4 \pi} \iint_{A} 4 \Delta l \frac{A_{1} A_{2}\left[A_{1} \partial_{r} A_{2}-\partial_{r} A_{1} A_{2}\right]}{\left[A_{1}^{2}+A_{2}^{2}\right]^{2}} d r d \phi \\
& =\frac{\Delta l}{2 \pi} \int_{0}^{2 \pi} d \phi \int_{r_{1}}^{r_{2}} \partial_{r} \frac{-A_{1}^{2}}{A_{1}^{2}+A_{2}^{2}} d r \\
& =\Delta l\left[\frac{A_{1}\left(r_{1}\right)^{2}}{A_{1}\left(r_{1}\right)^{2}+A_{2}\left(r_{1}\right)^{2}}-\frac{A_{1}\left(r_{2}\right)^{2}}{A_{1}\left(r_{2}\right)^{2}+A_{2}\left(r_{2}\right)^{2}}\right] .
\end{aligned}
$$

Therefore, the skyrmion number $N_{\text {sk }}$ is proportional to the difference in OAM $\Delta l$, and exactly equals $\Delta l$ when the spin states are $\left|s_{1}\right\rangle$ at $r_{1}$ (or $A_{2}\left(r_{1}\right)=0$ ) and $\left|s_{2}\right\rangle$ at $r_{2}$ (or $\left.A_{1}\left(r_{2}\right)=0\right) . N_{\text {sk }}$ does not depend on the phase offset $\gamma(r)$. Since the mode profile is known to depend on $|l|, r_{1}$ and $r_{2}$ satisfying such a condition often exist under the condition of $\left|l_{2}\right| \neq\left|l_{1}\right|$. For analytically calculating the skyrmion number of an FP beam generated by our microring cavity, we assumed the Bessel-like far-field distribution discussed in Eq. (A11) and obtained

$$
\begin{aligned}
N_{\text {sk }}(\theta)= & \Delta l \frac{J_{\left|l_{1}\right|}(0)^{2}}{J_{\left|l_{1}\right|}(0)^{2}+J_{\left|l_{2}\right|}(0)^{2}} \\
& -\Delta l \frac{J_{\left|l_{1}\right|}\left(k d_{1} \sin \theta\right)^{2}}{J_{\left|l_{1}\right|}\left(k d_{1} \sin \theta\right)^{2}+J_{\left|l_{2}\right|}\left(k d_{2} \sin \theta\right)^{2}},
\end{aligned}
$$

where $d_{1}\left(d_{2}\right)$ is the radius of the angular grating that diffracts a beam in an angular momentum state of $|s, l\rangle=$ $\left|+1, l_{1}\right\rangle\left(\left|-1, l_{2}\right\rangle\right)$.

\section{APPENDIX D: DETAILED FAR-FIELD PROFILE OF AN FP BEAM}

In this section, we take a closer look to the field pattern of an FP beam. We consider an FP beam with $N_{\mathrm{sk}}=+1$, which is synthesized from two beams in the angular momentum states of $|s, l\rangle=|+1,0\rangle$ and $|-1,+1\rangle$. Each beam in the different angular momentum state can be independently generated by solely placing a single angular grating on the microring. The far-field pattern of such a beam generated by the single grating can be described by a Bessel function, as derived in a previous section. Figure 7(a) shows results obtained with the toy model. The far-field pattern is overlaid with the plot of spin distribution. The top panel of the right bottom inset of Fig. 7(a) shows far-field patterns diffracted from individual angular gratings. It is obvious that the two beams (colored in red and blue) generated from the different angular gratings have different positions of intensity zero, at which complete spin flips occur in the superposed beam (light green). The spin flip induces the oscillation of $N_{\text {sk }}$ as computed in the bottom panel in the right bottom inset of Fig. 7(a). The behavior of spin flip is visualized in the left bottom inset. The structure that skyrmions and antiskyrmions appear alternately is known as a skyrmion multiplex [44]. In the skyrmionic structure, one can find a variety of skyrmionic structures, such as skyrmionium, skyrmion, and meron. These states may be obtained by spatially filtering the FP beam carrying the skyrmion multiplex.

Figure 7(b) shows the same set of results calculated using the FDTD method. Overall, the FDTD simulations reproduce the results obtained by the toy model in particular when $\theta$ is small. One noticeable deviation can be found in the behavior of $N_{\text {sk }}$, which does not return to zero when varying $\theta$. This is due to the imperfection in the spin flips at the zero field intensity points. In the case of FDTD simulations, unspecified background radiation scrambles the spin state where the diffracted signal is weak. The behavior of $N_{\text {sk }}$ also significantly deviates when $\theta$ is large, where the approximation used in the toy model does not stand. The use of a microring with a larger radius would be helpful for focusing the beam power to the region with small $\theta$ and thereby for generating more ideal FP beams.

\section{APPENDIX E: SIMULATING BEAMS WITH $N_{\mathrm{sk}}= \pm 2, \pm 3$}

In this section, we discuss the generation of FP beams with $N_{\text {sk }}= \pm 2$ and \pm 3 to further confirm the generality of our scheme. Similar to the cases discussed in Fig. 3 for the beam with $N_{\mathrm{sk}}=+1$ and in Fig. 4 for $N_{\mathrm{sk}}=-1$, we properly change $g$ for the grating emitting $s=-1$ beam to control its 

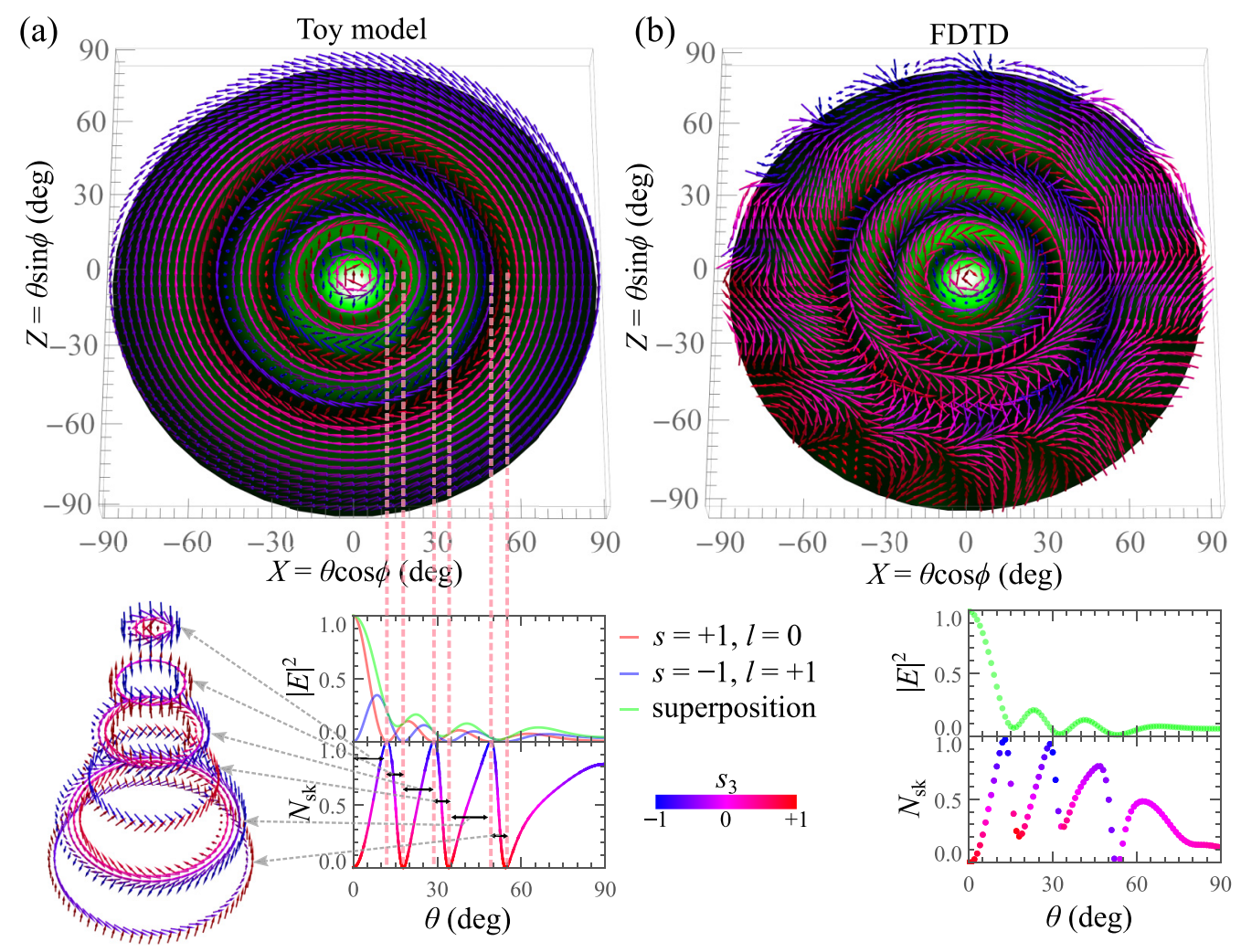

FIG. 7. Far-field intensity and spin distribution of an FP beam with $N_{\mathrm{sk}}=+1$, which is synthesized from two beams in the angular momentum states of $|s, l\rangle=|+1,0\rangle$ and $|-1,+1\rangle$. (a) Results by the toy model. The right bottom insets show slices of field intensity and evolution of $N_{\mathrm{sk}}$. The left bottom inset shows spin distributions between neighbor oscillations of $N_{\mathrm{sk}}$. Each of the spin distribution constitutes a skyrmion or an antiskyrmion with $\left|N_{\mathrm{sk}}\right|=1$. (b) Results from the FDTD calculations.

$l$, while keeping the same $g$ for that emitting $s=+1$ beam. In this way, $\Delta l$ is controlled so as to generate the beam with a target $N_{\text {sk }}$. For example, by changing $g$ of an $s=-1$ angular grating to 23 , a beam in a state of $|s, l\rangle=|-1,+2\rangle$ will be radiated from the grating, resulting in an FP beam with an $N_{\text {sk }}$ of +2 , as shown in Fig. 8(a). Figures 8(b)-8(d) show that FP beams with an $N_{\mathrm{sk}}=+3(-2,-3)$ can also be generated by changing $g$ of $s=-1$ angular grating to $22(27,28)$ so that a beam in a state of $|s, l\rangle=|-1,+3(-2,-3)\rangle$ will be radiated from the angular grating.

\section{APPENDIX F: CONTROLLING THE HELICITY OF A SKYRMION}

Our scheme is also suitable for generating different types of skrymions other than the Bloch type demonstrated in Fig. 3. The type of skyrmion is determined by the helicity $\gamma$ [48], which corresponds to the phase offset between two orthogonal spin states. The phase offset for the case of the angular gratings that are aligned at $\phi=0$ as depicted in the left panel of Fig. 9 is $\gamma=\exp [-i \Delta|l| \pi / 2]$, which can be easily estimated using Eq. (A11). In this case, $\gamma$ becomes $-\pi / 2$ for the beam with $N_{\text {sk }}(=\Delta l)=+1$, resulting in a Bloch-type skyrmion shown in the left panel in Fig. 9. By rotating one of the two angular gratings by a certain angle $\left(\phi_{0}\right)$ in the azimuth direction, the diffracted beams gain an additional relative phase offset of $\exp \left[i g \phi_{0}\right]$, where $g$ is the number of grating elements. Therefore, by rotating the $s=-1$ angular grating by $\frac{\pi / 2}{g(=24)}=3.75^{\circ}, \gamma$ becomes 0 and we obtain a Néel-type skyrmion beam, as shown in the right panel in Fig. 9.

\section{APPENDIX G: ROBUST GENERATION OF A BEAM WITH A LARGE $N_{\text {sk }}$}

We observed that $N_{\mathrm{sk}}$ did not reach the designed value of -5 near $\theta=13^{\circ}$ for the device discussed in Fig. 4(f). This discrepancy arises from the weak signal of the OAM beam with a large $l$ at low $\theta$. The weak signal was obscured by background noise straying in the simulator, resulting in the unexpected behavior of $N_{\mathrm{sk}}$. The weak signal even at a moderate $\theta$ stems from the donut shape of the OAM beam, the diameter of which becomes larger as $l$ increases. The background noise could originate from imperfections in the isolated excitation of the target WGM and the radiation from the microring cavity induced by anything other than the grating. Indeed, with the FDTD method, we have detected the presence of the radiation from the other WGM that was unintentionally excited in the simulator. The discrepancy of the FDTD simulations from the toy model can also be caused by the finite size of the grating element, which prevents generating pure circular polarization upon scattering and by the imperfections in the placement of grating elements from the ideal positions. The fine tuning of the simulator model to resolve these issues would lead to a good agreement between the simulator and the toy model, though such an approach is cumbersome and obviously not smart. 
(a)

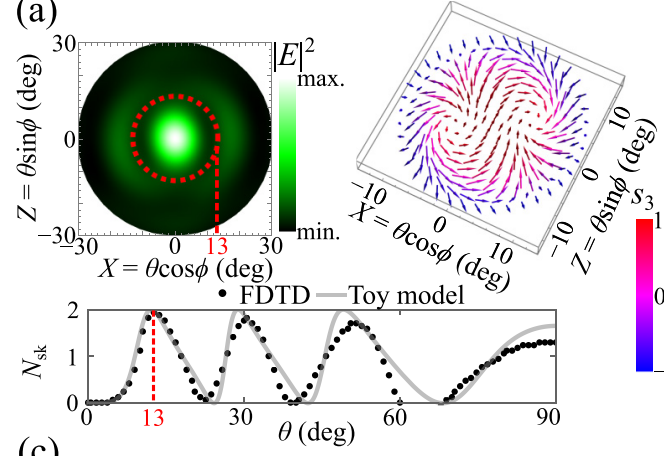

(c)

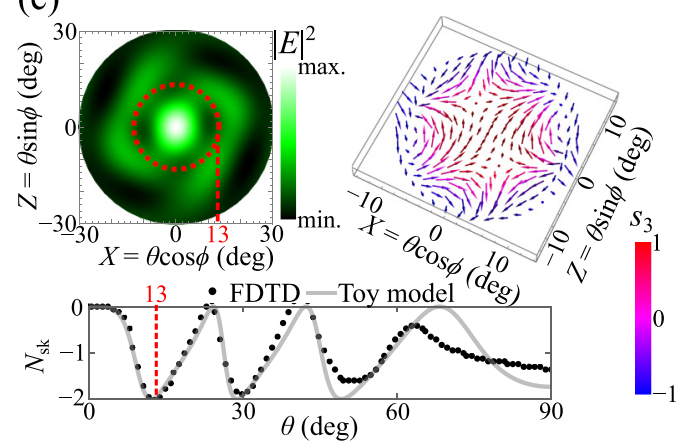

(b)

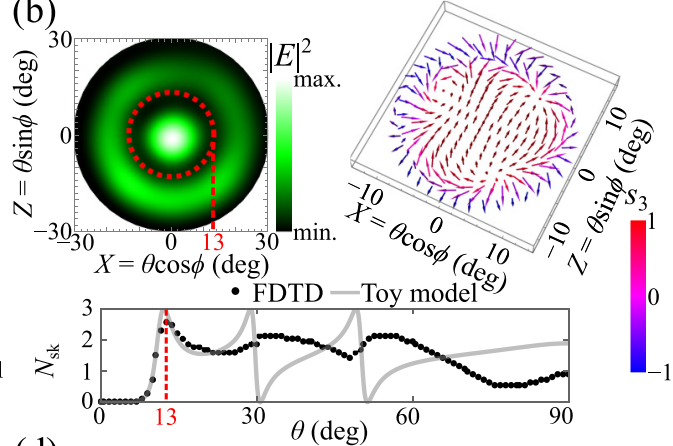

(d)

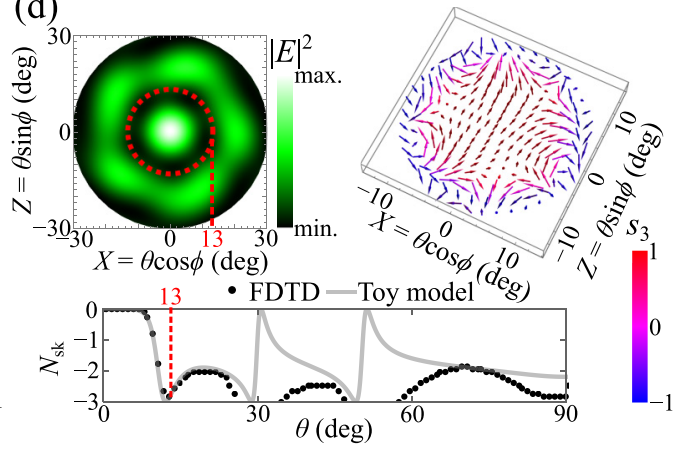

FIG. 8. Generation of FP beams with $N_{\text {sk }}= \pm 2$ and \pm 3 . (a-d) Same set of plots as Figs. 4(a)-4(c) but for the beams with a designed $N_{\text {sk }}$ of (a) +2 , (b) +3 , (c) -2 , and (d) -3 .

A simpler method to improve the simulator model is to reconsider the numbers of grating elements $g$ on both angular gratings. In the main text, we have modified only one of the two gratings when controlling the $N_{\mathrm{sk}}$ of a generated beam. This leads to a large discrepancy among the beam diameters of the beams radiated from the two gratings and thus to the low

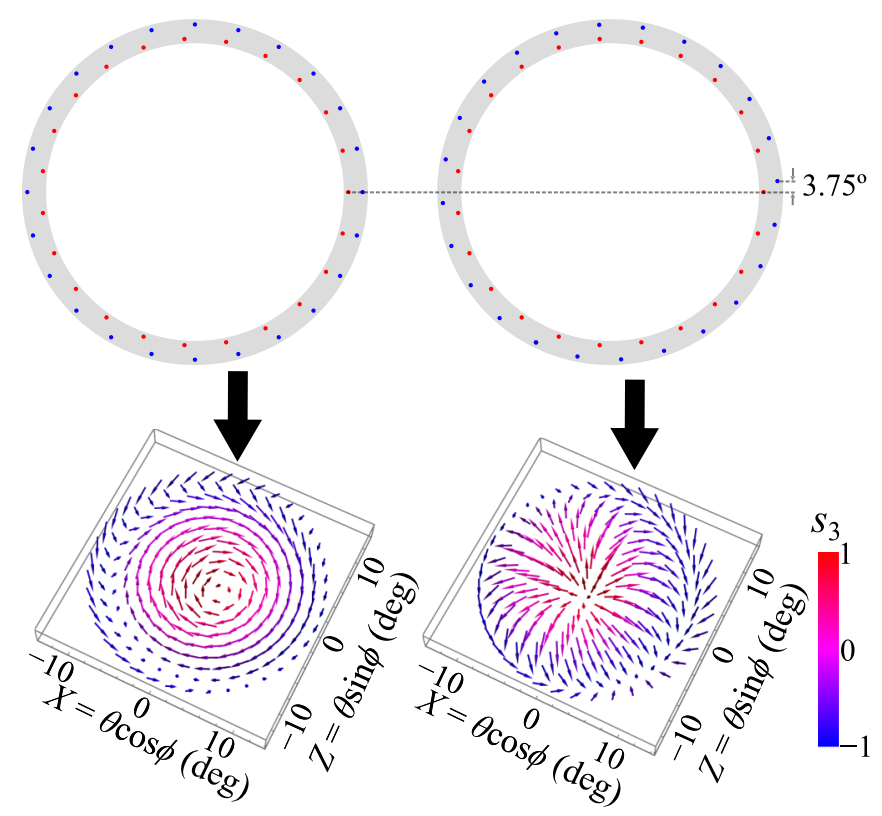

FIG. 9. Schematic showing a method to control the helicity of skyrmion. The rotation of one of the angular gratings (blue dotted) in the azimuthal direction by $3.75^{\circ}$ transforms a Bloch-type skymion beam (left) to a Néel-type one (right).

spatial overlap between them. Meanwhile, the spatial overlap can be increased by minimizing the difference in the $l(\Delta|l|)$ of the two beams. In this way, the area occupied by the weak signal will be minimized and the effect of the background will be mitigated. Here we consider the generation of orthogonally spin-polarized two beams with $l=+1$ and -4 . While $\Delta|l|$ is reduced to $3, \Delta l$ is still -5 such that we can expect the generation of a skyrmion beam with $N_{\mathrm{sk}}$ of -5 through the superposition of the two beams in the states of $|-1,-4\rangle$ and $|+1,+1\rangle$. The former (latter) state can be generated from an $s=-1(s=+1)$ grating with a grating number of 29 (22) by

(a)

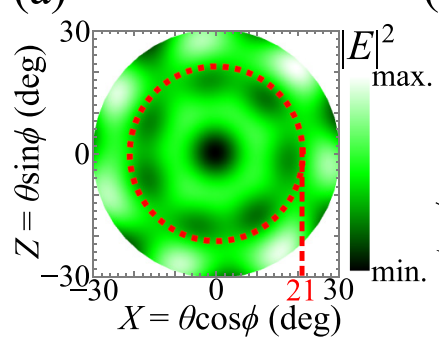

(c)

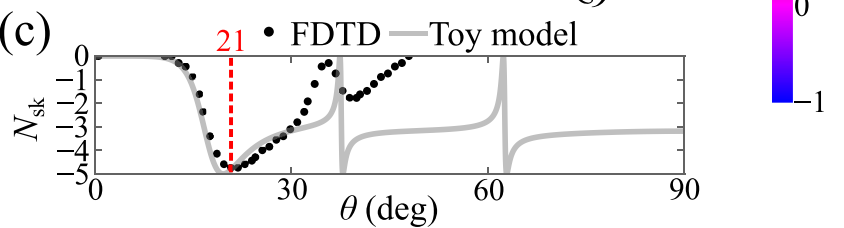

FIG. 10. Generation of an FP beam with $N_{\text {sk }}=-5$ by synthesizing two optical vortices with non-zero OAMs. (a) Projected far-field intensity profile. (b) Stokes vector field. (c) Calculated skyrmion number versus elevation angle.

(b)

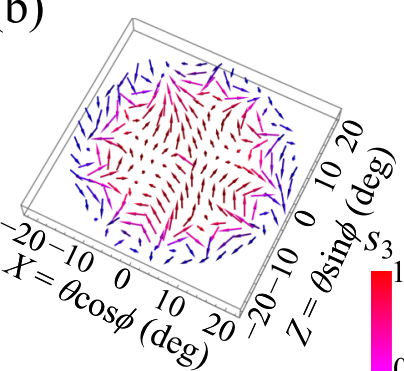


exciting a WGM with the azimuthal order of 24. Figure 10 shows the corresponding results, which exhibit a good agreement with the results of the toy model in the range $\theta \leqslant 30^{\circ}$. We note that some discrepancy between the simulation and the toy model remains near the beam center at low $\theta$. This originates from the weak signal stemming from the donut shape of the two beams.

Another strategy for better generating a skyrmion beam with a large $N_{\mathrm{sk}}$ is to modify the microring cavity structure itself. The beam diameter becomes smaller with increasing the size of the microring. Therefore, we could use two different microrings coaxially arranged on the same chip for separately generating the beams with different $l$, while matching the diameters of the two beams. In this way, even using the beams with $l=0$ and a large $l$, we will be able to maintain a large spatial overlap between the two beams without having a low signal region near the beam cross-section center. We note that the coaxial two microrings could be replaced with a single microring with a wider waveguide width.
[1] L. Allen, M. W. Beijersbergen, R. J. C. Spreeuw, and J. P. Woerdman, Orbital angular momentum of light and the transformation of Laguerre-Gaussian laser modes, Phys. Rev. A 45, 8185 (1992).

[2] N. B. Simpson, K. Dholakia, L. Allen, and M. J. Padgett, Mechanical equivalence of spin and orbital angular momentum of light: An optical spanner, Opt. Lett. 22, 52 (1997).

[3] G. Milione, H. I. Sztul, D. A. Nolan, and R. R. Alfano, HigherOrder Poincaré Sphere, Stokes Parameters, and the Angular Momentum of Light, Phys. Rev. Lett. 107, 053601 (2011).

[4] X. Yi, Y. Liu, X. Ling, X. Zhou, Y. Ke, H. Luo, S. Wen, and D. Fan, Hybrid-order Poincaré sphere, Phys. Rev. A 91, 023801 (2015).

[5] D. Naidoo, F. S. Roux, A. Dudley, I. Litvin, B. Piccirillo, L. Marrucci, and A. Forbes, Controlled generation of higher-order Poincaré sphere beams from a laser, Nat. Photonics 10, 327 (2016).

[6] Z. Liu, Y. Liu, Y. Ke, Y. Liu, W. Shu, H. Luo, and S. Wen, Generation of arbitrary vector vortex beams on hybrid-order Poincaré sphere, Photonics Res. 5, 15 (2017).

[7] A. M. Beckley, T. G. Brown, and M. A. Alonso, Full Poincaré beams, Opt. Express 18, 10777 (2010).

[8] J. C. Suárez-Bermejo, J. C. G. de Sande, M. Santarsiero, and G. Piquero, Mueller matrix polarimetry using full Poincaré beams, Optics Lasers Eng. 122, 134 (2019).

[9] L.-G. Wang, Optical forces on submicron particles induced by full Poincaré beams, Opt. Express 20, 20814 (2012).

[10] W. Zhu, V. Shvedov, W. She, and W. Krolikowski, Transverse spin angular momentum of tightly focused full Poincaré beams, Opt. Express 23, 34029 (2015).

[11] B. Khajavi and E. J. Galvez, High-order disclinations in spacevariant polarization, J. Opt. 18, 084003 (2016).

[12] E. Otte, C. Alpmann, and C. Denz, Higher-order polarization singularities in tailored vector beams, J. Opt. 18, 074012 (2016).

[13] X. Ling, X. Yi, Z. Dai, Y. Wang, and L. Chen, Characterization and manipulation of full Poincaré beams on the hybrid Poincaré sphere, J. Opt. Soc. Am. B 33, 2172 (2016).

[14] J. Wang, L. Wang, and Y. Xin, Generation of full Poincaré beams on arbitrary order Poincaré sphere, Curr. Opt. Photonics 1, 631 (2017).

[15] E. J. Galvez, S. Khadka, W. H. Schubert, and S. Nomoto, Poincaré-beam patterns produced by nonseparable superpositions of Laguerre-Gauss and polarization modes of light, Appl. Opt. 51, 2925 (2012).
[16] F. Cardano, E. Karimi, L. Marrucci, C. de Lisio, and E. Santamato, Generation and dynamics of optical beams with polarization singularities, Opt. Express 21, 8815 (2013).

[17] V. Shvedov, P. Karpinski, Y. Sheng, X. Chen, W. Zhu, W. Krolikowski, and C. Hnatovsky, Visualizing polarization singularities in Bessel-Poincaré beams, Opt. Express 23, 12444 (2015).

[18] D. Lopez-Mago, On the overall polarisation properties of Poincaré beams, J. Opt. 21, 115605 (2019).

[19] S. Donati, L. Dominici, G. Dagvadorj, D. Ballarini, M. De Giorgi, A. Bramati, G. Gigli, Y. G. Rubo, M. H. Szymańska, and D. Sanvitto, Twist of generalized skyrmions and spin vortices in a polariton superfluid, Proc. Natl. Acad. Sci. USA 113, 14926 (2016).

[20] S. Gao, F. C. Speirits, F. Castellucci, S. Franke-Arnold, S. M. Barnett, and J. B. Götte, Paraxial skyrmionic beams, Phys. Rev. A 102, 053513 (2020).

[21] X. Z. Yu, Y. Onose, N. Kanazawa, J. H. Park, J. H. Han, Y. Matsui, N. Nagaosa, and Y. Tokura, Real-space observation of a two-dimensional skyrmion crystal, Nature (London) 465, 901 (2010).

[22] J.-y. Choi, W. J. Kwon, and Y.-i. Shin, Observation of Topologically Stable 2D Skyrmions in an Antiferromagnetic Spinor Bose-Einstein Condensate, Phys. Rev. Lett. 108, 035301 (2012).

[23] J.-i. Fukuda and S. Zumer, Quasi-two-dimensional skyrmion lattices in a chiral nematic liquid crystal, Nat. Commun. 2, 246 (2011).

[24] P. Cilibrizzi, H. Sigurdsson, T. C. H. Liew, H. Ohadi, A. Askitopoulos, S. Brodbeck, C. Schneider, I. A. Shelykh, S. Höfling, J. Ruostekoski, and P. Lagoudakis, Half-skyrmion spin textures in polariton microcavities, Phys. Rev. B 94, 045315 (2016).

[25] S. Tsesses, E. Ostrovsky, K. Cohen, B. Gjonaj, N. H. Lindner, and G. Bartal, Optical skyrmion lattice in evanescent electromagnetic fields, Science 361, 993 (2018).

[26] L. Du, A. Yang, A. V. Zayats, and X. Yuan, Deepsubwavelength features of photonic skyrmions in a confined electromagnetic field with orbital angular momentum, Nat. Phys. 15, 650 (2019).

[27] C. Bai, J. Chen, Y. Zhang, D. Zhang, and Q. Zhan, Dynamic tailoring of an optical skyrmion lattice in surface plasmon polaritons, Opt. Express 28, 10320 (2020). 
[28] C. Guo, M. Xiao, Y. Guo, L. Yuan, and S. Fan, Meron Spin Textures in Momentum Space, Phys. Rev. Lett. 124, 106103 (2020).

[29] K. Wang, X. Qiu, L. Xiao, X. Zhan, Z. Bian, B. C. Sanders, W. Yi, and P. Xue, Observation of emergent momentum-time skyrmions in parity-time-symmetric non-unitary quench dynamics, Nat. Commun. 10, 2293 (2019).

[30] J. Wätzel and J. Berakdar, Topological light fields for highly non-linear charge quantum dynamics and high harmonic generation, Opt. Express 28, 19469 (2020).

[31] T. Bauer, P. Banzer, E. Karimi, S. Orlov, A. Rubano, L. Marrucci, E. Santamato, R. W. Boyd, and G. Leuchs, Observation of optical polarization Möbius strips, Science 347, 964 (2015).

[32] I. J. Luxmoore, N. A. Wasley, A. J. Ramsay, A. C. T. Thijssen, R. Oulton, M. Hugues, S. Kasture, V. G. Achanta, A. M. Fox, and M. S. Skolnick, Interfacing Spins an an InGaAs Quantum Dot to a Semiconductor Waveguide Circuit Using Emitted Photons, Phys. Rev. Lett. 110, 037402 (2013).

[33] I. Söllner, S. Mahmoodian, S. L. Hansen, L. Midolo, A. Javadi, G. Kirsanske, T. Pregnolato, H. El-Ella, E. H. Lee, J. D. Song et al., Deterministic photon-emitter coupling in chiral photonic circuits, Nat. Nanotechnol. 10, 775 (2015).

[34] R. J. Coles, D. M. Price, J. E. Dixon, B. Royall, E. Clarke, P. Kok, M. S. Skolnick, A. M. Fox, and M. N. Makhonin, Chirality of nanophotonic waveguide with embedded quantum emitter for unidirectional spin transfer, Nat. Commun. 7, 11183 (2016).

[35] X. Cai, J. Wang, M. J. Strain, B. Johnson-Morris, J. Zhu, M. Sorel, J. L. O'Brien, M. G. Thompson, and S. Yu, Integrated compact optical vortex beam emitters, Science 338, 363 (2012).

[36] Q. Xiao, C. Klitis, S. Li, Y. Chen, X. Cai, M. Sorel, and S. $\mathrm{Yu}$, Generation of photonic orbital angular momentum superposition states using vortex beam emitters with superimposed gratings, Opt. Express 24, 3168 (2016).
[37] Z. Shao, J. Zhu, Y. Chen, Y. Zhang, and S. Yu, Spin-orbit interaction of light induced by transverse spin angular momentum engineering, Nat. Commun. 9, 926 (2018).

[38] M. Martinelli and P. Martelli, Polarization, mirrors, and reciprocity: Birefringence and its compensation in optical retracing circuits, Adv. Opt. Photonics 9, 129 (2017).

[39] R. Ozawa, S. Hayami, and Y. Motome, Zero-Field Skyrmions with a High Topological Number in Itinerant Magnets, Phys. Rev. Lett. 118, 147205 (2017).

[40] X. Zhang, Y. Zhou, and M. Ezawa, High-topological-number magnetic skyrmions and topologically protected dissipative structure, Phys. Rev. B 93, 024415 (2016).

[41] R. Yu, Y. Xin, Q. Zhao, Y. Shao, and Y. Chen, Exceptional polarization structures near the C-lines in diffracted near fields, J. Opt. Soc. Am. A 32, 1468 (2015).

[42] T. Bauer, M. Neugebauer, G. Leuchs, and P. Banzer, Optical Polarization Möbius Strips and Points of Purely Transverse Spin Density, Phys. Rev. Lett. 117, 013601 (2016).

[43] A. Garcia-Etxarri, Optical polarization Möbius strips on alldielectric optical scatterers, ACS Photonics 4, 1159 (2017).

[44] H. Fujita and M. Sato, Ultrafast generation of skyrmionic defects with vortex beams: Printing laser profiles on magnets, Phys. Rev. B 95, 054421 (2017).

[45] X. Z. Yu, W. Koshibae, Y. Tokunaga, K. Shibata, Y. Taguchi, N. Nagaosa, and Y. Tokura, Transformation between meron and skyrmion topological spin textures in a chiral magnet, Nature (London) 564, 95 (2018).

[46] A. S. Ostrovsky, C. Rickenstorff-Parrao, and V. Arrizón, Generation of the "perfect" optical vortex using a liquid-crystal spatial light modulator, Opt. Lett. 38, 534 (2013).

[47] P. Vaity and L. Rusch, Perfect vortex beam: Fourier transformation of a Bessel beam, Opt. Lett. 40, 597 (2015).

[48] N. Nagaosa and Y. Tokura, Topological properties and dynamics of magnetic skyrmions, Nat. Nanotechnol. 8, 899 (2013). 